\title{
Joint Mechanisms of the Collar-Connection-Element
}

\author{
Arne Mann and Peter Groche
}

\begin{abstract}
The society demand for a responsible interaction with the environment and resources has increased over the last decades. Therefore, lightweight design has been the focus in research and development for several years. There are different strategies for obtaining the aims of weight reduction and efficient material usage. New lightweight materials and designs underlie a difficult economic introduction in existing manufacturing processes and products. This paper introduces the design for a new connection element. The collar-connection element is based on the collar forming process. It consists of two collars having different diameters fitting into each other. A mechanical joint connects both collars and provides a load-transmission between the sheet metals. Hereby, the collar-connection element enables the design of an all-metal-sandwich structure possessing a high specific stiffness and strength. This paper presents the functionality of the collar-connection element and investigates the acting joint mechanisms.
\end{abstract}

Index Term-Collar forming, connection element, joining mechanism, joining by forming.

\section{INTRODUCTION}

Forming technologies are a highly efficient and economical way of manufacturing. Low costs, due to the use of efficient mass production processes, open the access to many products for the majority of the population. However, lightweight design and materials fail at an economic implementation in manufacturing processes in many cases. This paper introduces the collar-connection element (Fig. 1). The collar-connection element can be utilized to connect two sheets. The application of the elements in a two-dimensional structure across the surface of the sheets results in a stiff structure with an adjusted sheet distance. This structure can be used for the design of the core material for a sandwich structure. According to present research, sandwich structures are classified based on their core design. In this scope, the present all-metal sandwich structure design using the collar-connection element can be categorized to the structural cores as a punctual supporting core element [1] or as a corrugated or web core material [2].

Forming processes have been used for the production of sandwich cores in several studies. WADLEY uses a press

Manuscript received Febrary 9, 2017; revised June 20, 2017. This paper is supported by the German Federation of Industrial Research Associations (AiF) with means of the Federal Ministry of Economic Affairs and Energy (BMWi) on the basis of a decision by the German Bundestag.

The authors are with the Institute for Production Engineering and Forming Machines at Technische Universität Darmstadt, Germany (e-mail: mann@ ptu.tu-darmstadt.de, groche@ ptu.tu-darmstadt.de). brake process to bend a tetrahedral truss core [3]. BESSE uses a stamping process for manufacturing a corrugated core layer [4]. LÄMSÄ [5] and JÄRVENPÄÄ [6] use a press brake process to produce a corrugated core. All approaches have a metallurgical bond creation in common. However, the metallurgical joint creation requires higher efforts in preparation and cycle time. In contrast, the collar-connection element is based on collar forming processes being a highly productive forming process, which is investigated extensively. Therefore, the new connection element promises an economical manufacturing process with adequate cycle times compared to collar forming. The collar-connection element is based on a mechanical joint. Two collars with different diameters are formed, so that they fit into each other. The joint creation between both sheets is realized by a radial expansion of the collars. MORI et al. [7] and GROCHE et al. [8] sum up that many forming technologies are utilized for a bond creation by plastic deformation. The bond creation is separated in mechanical joining and metallurgical joining. The mechanical joints can be subdivided in form-closed joints and force-closed joints. The following joint creation of both collars is realized by a mechanical joint in a combination of a form-closed and force-closed joint. This paper presents the design of the connection element and investigates the resulting bonding force both experimentally and numerically. The numerical analysis identifies the relation between certain geometry and tool parameters and the load carrying capacity. Finally, the numerical simulation is validated by comparing it to the results of an experiment.

\section{DESIGN OF THE COLLAR-CONNECTION ELEMENT}

Fig. 1 presents the collar-connection element applied to two sheets in a side view (i) and in a cross section (ii). The collar-connection element consists of two collars formed in separate collar forming processes. The collar diameters are designed in such a way that both collars fit into each other. The connection element must meet the requirement of an axial tensile and compression force transmission. For the transmission of the compression force, the outer collar $a$ is positioned with its collar tip on the collar diameter $r_{b, c}$ of the inner collar $b$ (Fig. 1 iii). For a tensile load transmission, a connection between the outer collar $a$ and the inner collar $b$ has to be established. Therefore, a punch moves coaxially in the direction of the outer collar $b$ into the collars. The conical characteristic of the punch is defined by the angle $\alpha$. The radial displacement of the inner and outer collar induced by the conical punch sets a form-closed joint. The small punch angle $\alpha$ suggests that friction has a major influence on the axial tensile strength (pull-out strength) $S_{a x}$. Furthermore, a superimposed force-closed fit exists due to the induced surface pressure between inner and outer collar. The surface 
pressure between the collars results from the radial displacement during the joint creation and a relative axial displacement of the collars during load exposure. (i)

(ii)

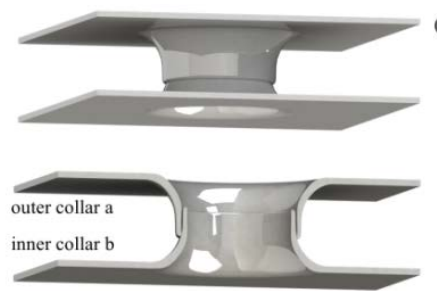

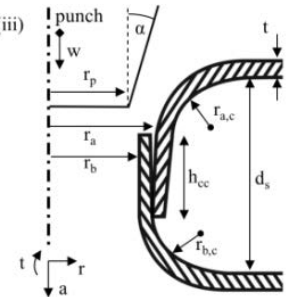

Fig. 1. Side view onto the collar connection element (i), cross section (ii), schematic drawing (iii).

Both collars are formed separately prior to the joining process (Fig. 1. iii). The overlapping height $h_{c c}$ is expected to have a major influence on the axial pull-out strength of the connection element. For a maximum collar height, the gap between the collar forming tools was chosen to be 0.8 times the sheet thickness. As a result, there are higher collars with a cylindrical geometry compared to a gap size equal to the sheet thickness. The collar rounding radii $r_{a, c}$ and $r_{b, c}$ with the overlap of both collars add up to the sheet distance $d_{s}$. The collar rounding radii are set to $r_{a, c}$ and $r_{b, c}=4 \mathrm{~mm}$. The collar radii are $r_{a}=7.8 \mathrm{~mm}$ and $r_{b}=7.1 \mathrm{~mm}$, respectively. This results in an overlapping height $h_{c c}=3 \mathrm{~mm}$ and a sheet distance $d_{S}=11 \mathrm{~mm}$. For this study, the steel grade DC01 with a sheet thickness $t=0.88 \mathrm{~mm}$ is employed. A geometrical analysis of the collars has shown that the sheet thickness is reduced to $t=0.62 \mathrm{~mm}$ at the collar tip due to the collar forming process. For the numerical analyses, material properties are recorded in standardized tensile tests with flat bar tension specimen. The process window for the collar forming process was elaborated by a hole expansion test using drilled holes as initial condition.
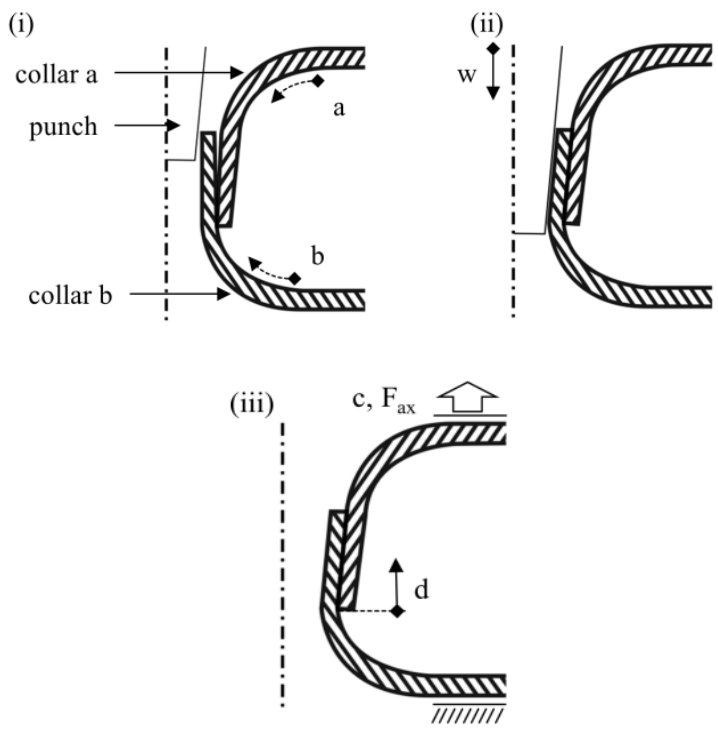

Fig. 2. Form-closed joint manufacturing process, simplified axial symmetric view.

Fig. 2 shows the manufacturing process of the form-closed joint. For the process design, a finite element model was built. In the model, the collars $a$ and $b$ are positioned coaxially (i). The tip of collar $a$ is supported by the collar rounding radius of collar $b$. In a first step, a conical punch modelled as an analytical rigid body moves coaxially in the collar-connection element in direction of the outer collar $a$ (ii). The punch displacement $\mathrm{w}$ stops after the form-closed joint has been generated. The pull-out strength $S_{a x}$ is used to evaluate the connection strength. In the simulation, the outer collar $a$ is fixed using boundary conditions and the pull-out force $F_{a x}$ is recorded during the extraction process being defined by an axial displacement (iii). The finite element model is designed axially symmetric using the software Simulia Abaqus, version 6.14-1. The strain hardening due to the collar forming process is modeled by shifting the yield point of the material with increasing collar height. The mesh is built using axially symmetric, linear elements with reduced integration and hourglass control (CAX4R). The collars have three elements in thickness direction and an edge length of $0.2 \mathrm{~mm}$ in sheet direction. The model uses an implicit solver. The friction coefficient is adapted during the validation starting at a value of $\mu=0$. The FE model is used to investigate the punch angle $\alpha$ and the punch displacement $\mathrm{w}$ (Fig. 1 iii).

\section{FE RESULTS AND VALIDATION}

The finite element model was used to vary geometric parameters and to determine the maximum pull-out strength of the collar-connection element. The expanding process using a conical punch causes a combination of a form-closed and force-closed joint between both collars. Fig. 3 shows three extraction stages of the collar-connection element in the pull-out test. The data is extracted from the finite element simulation. The first stage shows the initial state at the beginning of the extraction. The second stage shows the collar-connection element when the pull-out strength $S_{a x}$ is acting. The third picture presents the separated collars. Fig. 4 shows the corresponding pull-out force-displacement diagram. The pull-out strength is reached at $c \approx 0.5 \mathrm{~mm}$.

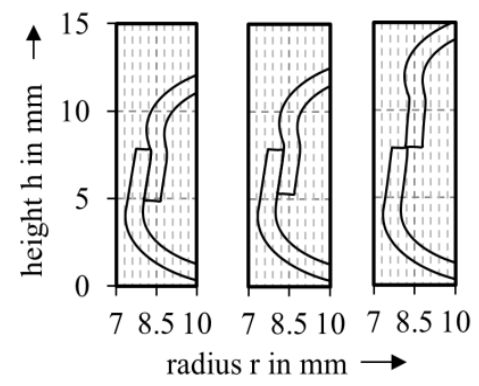

Fig. 3. Extraction stages of the collar-connection element taken from the simulation $\left(\alpha=6^{\circ}, w=4 \mathrm{~mm}, \mu=0.05\right.$; left to right: $c=0 \mathrm{~mm}, c=0.5 \mathrm{~mm}$, $c=3 \mathrm{~mm})$.

To separate both joining mechanisms in the following investigations, the influence of the punch angle $\alpha$ was examined in a frictionless simulation. The punch angle $\alpha$ was varied between $0^{\circ}$ and $8^{\circ}$. The punch angle $\alpha=0^{\circ}$ was implemented with a radial displacement of the punch. An increasing punch angle leads to a greater radial displacement of the collars. To obtain comparable results, the punch displacement $\mathrm{w}$ was calculated to reach a constant average radial displacement of the collars. Fig. 5 shows the maximum pull-out strength for each punch angle. Greater punch angles lead to an increasing radial deformation of the collars before they get separated. Therefore, the results fulfill the expectations. They show a linear correlation between punch 
angle and pull-out strength. The maximum pull-out strength $S_{a x}=571 \mathrm{~N}$ is reached at $\alpha=8^{\circ}$. For a practical implementation, punch angles greater than $6^{\circ}$ have to be investigated regarding their influence on a deformation of sheet $a$, since the increasing axial load can lead to a deformation of the sheet metal. In combination with the high radial load, the sheet metal shows a distortion in the plane sheet area outside the collar-connection element. For the validation, the punch angle $\alpha=6^{\circ}$ was realized.

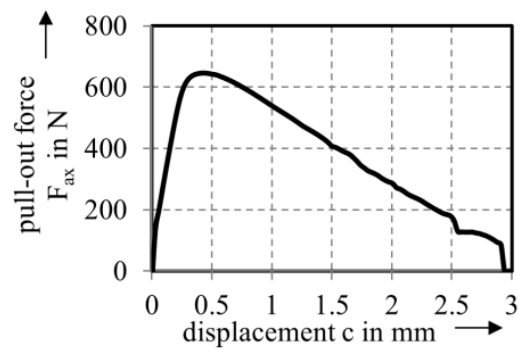

Fig. 4. Force-displacement diagram of the collar extraction simulation $(\alpha=$ $6^{\circ}, w=4 \mathrm{~mm}, \mu=0.05$ )

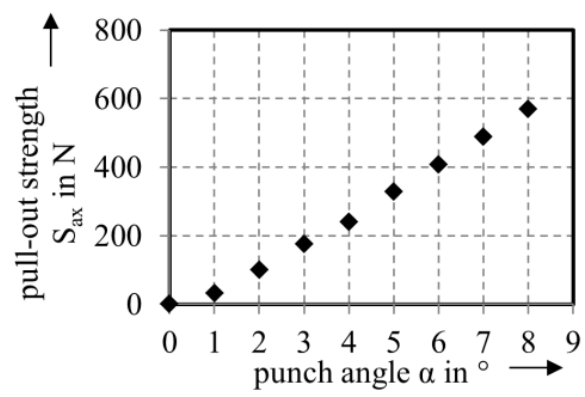

Fig. 5. Influence of the punch angle on the pull-out strength using constant radial displacement.

Fig. 6 shows the resulting surface pressure $p$ at the collar contact (see distance $d$ in Fig. 2 iii). The contact pressure can be seen as an indicator for a force-closed joint. The resulting contact pressure is partly caused by the increasing strain hardening induced during the collar forming process. Each collar is progressively strain-hardened with rising collar height, so that the hardened material properties are located in opposite directions of the collar-connection element. In the case that the more strain hardened material is positioned in the outer collar, the greater spring back of the outer collar in comparison to the inner collar results in a surface pressure between both collars. Accordingly, Fig. 6 shows the surface pressure at different stages. The step $w=4 \mathrm{~mm}$ shows the surface pressure at maximum punch displacement during the joining process. The surface pressure maximum is located at the inner collar tip. Here, the maximum radial displacement of the inner and outer collar is located, induced by the conical punch. The radial displacement due to the conical punch induces a bending load in the collars. The bending load is visible in the finite element simulation by a change from positive to negative radial stress levels in the collar rounding radii. Due to unloading, the spring back displacement induced by the bending load differs in value along path $a$ and path $b$ (Fig. 2 i). The spring back increases along the paths and reaches the maximum at the collar tips. Due to spring back, the remaining surface pressure in the collar-connection element is insignificant. At closer consideration, there is a different state of stress comparing the outer collar $a$ and inner collar $b$. The simulations show a higher level of superimposed compression in thickness direction in the inner collar at the point of maximum punch displacement. The superimposed compression in the inner collar is induced by the clamping between the punch and the outer collar. Regarding Mohr's circle, the superimposed compression reduces the tangential tensile stress necessary for reaching the yield criterion. Therefore, the inner collar can endure greater expanding ratios as stated by the hole expanding test. The positive influence of superimposed compression during collar forming is investigated in [9].

The step $c=0.0 \mathrm{~mm}$ in Fig. 6 shows the surface pressure at the beginning of the collar extraction. The low surface pressure level demonstrates the limited influence of a force-closed joint as a joining mechanism in the collar-connection element at the beginning of the extraction. The step $c=0.5 \mathrm{~mm}$ shows the surface pressure at the point of the pull-out strength. Due to the deformation of the collars during the extraction, significant contact pressures occur. While the collars are extracted further, the surface pressure is increasing and the distance $\mathrm{d}$ is decreasing. This results in lower pull-out force after $c=0.5 \mathrm{~mm}$.

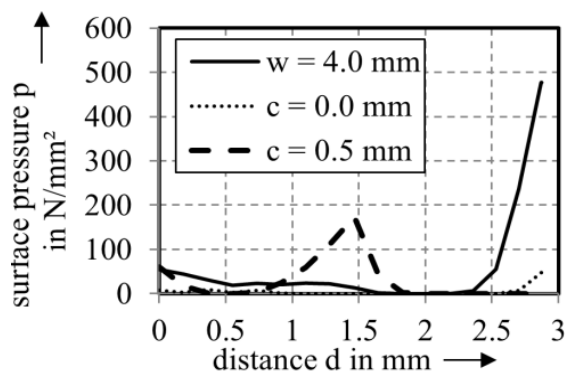

Fig. 6. Surface pressure between the collars, $\alpha=0^{\circ}, w=4 \mathrm{~mm}$.

In addition, the finite element model was used to determine the influence of the punch displacement on the pull-out strength of the collar-connection element. Therefore, the punch angle $\alpha=6^{\circ}$ was chosen with regard to the distortion in the plane sheet area. The friction coefficient was set to $\mu=0, \mu=0.05$ and $\mu=0.10$ for the experimental validation. Fig. 7 shows the results of the investigation. There is a linear correlation between the punch displacement and the axial strength up to $\mathrm{w}=4 \mathrm{~mm}$. Greater punch displacements lead to an increasing radial displacement of the collars followed by an increasing undercut between both collars. The pull-out strength increases. The slope of the straight line $\mu=0.05$ ( $\mu=$ $0.10)$ is steeper than for $\mu=0(\mu=0.05)$. This indicates an increasing influence of the contact pressure with increasing punch displacement. The punch displacement $w=4 \mathrm{~mm}$ results in the pull-out strength maximum. At the displacement $w=4 \mathrm{~mm}$, a complete surface contact of both collars is established. A greater punch displacement does not lead to an increasing surface contact. Therefore, the axial strength of the collar-connection element increases minimal with greater punch displacement. Furthermore, the process limit, elaborated by using the hole expansion test, is reached at the punch displacement $w=4 \mathrm{~mm}$.

Finally, the simulation data was validated. Therefore, a conical punch was used with $\alpha=6^{\circ}$. Greater punch angles could not be validated because of a following deformation of the sheets. The holes for the collar drawing process were drilled and the collars were formed by using a hydraulic press. The same hydraulic press was used to initiate the mechanical 
joint with a punch displacement $w=4 \mathrm{~mm}$. In the following, the collar-connection element was mounted in an axial tensile test machine and tested.

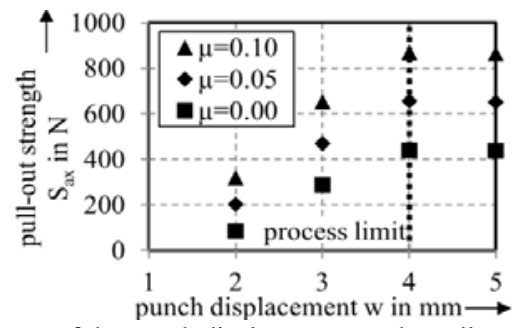

Fig. 7. Influence of the punch displacement on the pull-out strength.

Fig. 8 shows the results of the validation. The pull-out strength of the experiment corresponds with the finite element simulation by using the friction coefficient $\mu=0.05$. The given surfaces and the contact between two steel friction partners with expectedly oil contamination justify the assumption for the friction coefficient [10], S. 197.

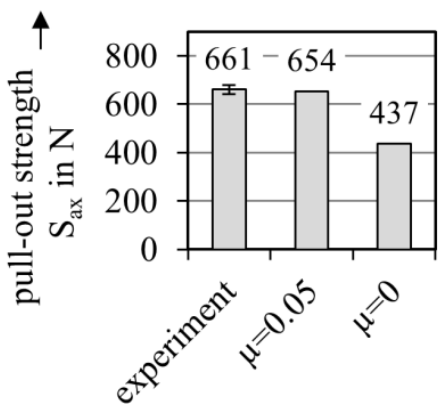

Fig. 8. Validation of the collar-connection element's axial strength.

\section{CONCLUSION}

This paper presented a new design of a connection element based on collar-forming. The collar-connection element is based on forming processes and it can, therefore, be manufactured efficiently. The influence of different process parameters was investigated using the finite element method. The present joint mechanisms of the mechanical joint were explained. Finally, the results of the simulation could be confirmed in the experimental validation. On the given basis, a sandwich structure based on the collar-connection element can be realized.

\section{REFERENCES}

[1] B. Klein, Leichtbau-Konstruktion Berechnungsgrundlagen und Gestaltung, Wiesbaden: Vieweg + Teubner, 2011.

[2] J. R. Vinson, "Sandwich structures," Appl. Mech. Rev., vol. 54, no. 3, pp. 201-214, May 2001.

[3] H. N. G. Wadley, N. A. Fleck, and A. G. Evans, "Fabrication and structural performance of periodic cellular metal sandwich structures," Composites Science and Technology, vol. 63, no. 16, pp. 2331-2343, Dec. 2003.

[4] C. C. Besse and D. Mohr, "Plasticity of formable all-metal sandwich sheets: Virtual experiments and constitutive modeling," International Journal of Solids and Structures, vol. 49, no. 19-20, pp. 2863-2880, Oct. 2012.

[5] J. Lämsä, A. Järvenpää, and K. Mäntyjärvi, "Designing and manufacturing of a flexible longitudinally laminated sandwich panel forming tool,” Key Engineering Materials, vol. 611-612, pp. 786-793, 2014.

[6] A. Järvenpää, J. Lämsä, M. Hietala, and K. Mäntyjärvi, “Mechanical properties of admin metal sandwich panel manufactured using longitudinally laminated forming tools,” Key Engineering Materials, vol. 611-612, pp. 781-785, 2014.

[7] K. Mori, N. Bay, L. Fratini, F. Micari, and A. E. Tekkaya, "Joining by plastic deformation,” CIRP Annals - Manufacturing Technology, vol. 62, no. 2, pp. 673-694, 2013.

[8] P. Groche, S. Wohletz, M. Brenneis, C. Pabst, and F. Resch, "Joining by forming - A review on joint mechanisms, applications and future trends," Journal of Materials Processing Technology, vol. 214, no. 10, pp. 1972-1994, Oct. 2014

[9] S. Schlagau, Verfahrensverbesserung beim Kragenziehen durch Überlagern von Druckspannungen, Technische Hochschule, 1988.

[10] H. Czichos and K. H. Habig, Eds., Tribologie-Handbuch, Wiesbaden: Springer Fachmedien Wiesbaden, 2015.

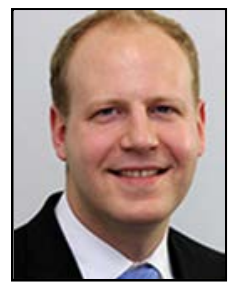

Arne Mann was born in Darmstadt, Germany in 1986 He earned a bachelor of science in mechanical and process engineering in 2011 and a master of science in mechanical and process engineering in 2013 at Technische Universität Darmstadt.

Currently, he is working on his doctoral thesis at the Institute for Production Engineering and Forming Machines, Technische Universität Darmstadt. 